\title{
Calibration of Chemical Applicators Used in Vegetables
}

\section{R.K. Sprenkel}

Calibration is the process of determining the amount of material applied per acre. Equipment for applying both liquid and dry material must be accurately calibrated for the pesticide to work properly and to avoid damaging or killing the crop if, in the case of herbicides, too much is applied. Also, since the label on a container of pesticide is considered to be a legal document, calibration of equipment ensures the product is being applied as specified on the label and that environmental contamination will be minimized. Some methods for calibration sprayers and dry material spreaders are described below.

\section{SPRAYER CALIBRATION}

The gallons per acre of diluted spray applied by a sprayer must be calculated to determine the number of acres that can be treated by a volume of spray. Once the acres that can be treated are known, the amount of pesticide formulation that should be added to the tank can be calculated by referring to the label on the pesticide container where it states the amount of chemical formulation recommended per acre.

Example. A calibration test showed a sprayer was applying 60 gallons per acre (GPA), how much pesticide formulation should be added when mixing 150 gallons of spray mixture if the label recommends 1 pound of formulation per acre? (See Equations 1,2 )

$$
\begin{aligned}
& \text { Acres treated by the spray }= \\
& \frac{\text { volume of spray }}{\text { gal/acre }}=\frac{150}{60}=2.5 \\
& \text { Pesticide required }= \\
& \frac{\text { acres }}{\text { tank }} \times \frac{\text { rate }}{\text { acre }}=2.5 \times 1=2.5 \mathrm{lbs}
\end{aligned}
$$

There are a number of ways to determine the gallons per acre of spray being applied. Some of the most common and versatile methods used are described here, but the method used is not as important as the results. If a grower uses another calibration method and successfully applies the pesticide in accordance with the label, then possibly that method should continue in use. However, it is recommended that growers should attempt to calibrate their sprayers as described below and compare the results to those obtained with their current method.
There are two types of calibration problems:

Type 1. The sprayer is already equipped with nozzles and the grower wants to determine the GPA being applied by the sprayer "as is".

Type 2. The grower wants to equip the sprayer with nozzles that will result in the sprayer applying the GPA desired by the grower.

The basic equation used to solve either problem is: (Equation 3)

$$
\mathrm{GPA}=\frac{\mathrm{GPM}}{\mathrm{ac} / \mathrm{min}}
$$

Where:

GPA = gallons per acre applied by the sprayer;

GPM = gallons per minute delivered by the sprayer nozzles;

$\mathrm{ac} / \mathrm{min}=$ the acres treated by the sprayer per minute

To determine the GPA of an already nozzled sprayer, the applicator must determine the gallons per minute delivered by the sprayer nozzles and the acres treated per minute.

\section{Determining GPM}

There are three commonly used methods for determining the GPM delivered by a sprayer: 1) a tank refill test; 2 ) use of volume graduations; and 3) the nozzle-flow test method, which is preferred.

\section{Tank Refill}

To determine GPM by the tank refill method, fill the sprayer to some well-marked, easily repeated level and run the sprayer for a measured period of time. Measure the amount of liquid needed to restore the level in the tank with the sprayer at the same location as it was when filled initially. The gallons per minute is found by dividing the gallons sprayed by the time in minutes.

Example. It takes 17 gallons to restore the initial level after running a sprayer for 3 minutes when using the pressure that will be used in the field. What is the GPM? (Equation 4 )

$$
\mathrm{GPM}=\frac{\text { gallons }}{\text { time }}=\frac{17}{3}=5.67 \frac{\mathrm{gal}}{\mathrm{min}}
$$




\section{Volume Graduations}

If the sprayer tank has visible volume graduations, GPM can be determined by running the sprayer until the level drops from one graduation to another and dividing by the time it takes for the level to drop. Use of tank graduations eliminates having to restore the initial level with a measured amount of water, which is often done by pouring 5 gallon buckets of water into the tank. Before using this method, the tank graduations should be checked for accuracy by pouring bucket loads of a known volume into the tank or using a water meter and comparing the known amount to that indicated by the graduations. If the level is significantly different from the graduations on the tank, the tank should be marked with new graduations.

Example. It takes 3 minutes and 44 seconds for the level of spray to drop from the 50 gallon graduation to the 25 gallon graduation. What is the GPM? (Equation 5 )

$$
\mathrm{GPM}=\frac{25}{3.73}=6.7
$$

Time has to be expressed in minutes, so 3 minutes and 44 seconds must be 3.73 minutes because 44 seconds is $44 / 60=0.73$ minutes.

\section{Nozzle Flow Test}

The preferred method for determining GPM is to catch the flow from each nozzle on the boom for approximately $1 / 2$ minute in a container graduated in ounces and determine the total ounces per minute by multiplying the sum of the individual nozzle flows by 2 (the sum would be multiplied by 4 , if the flow was caught for only $1 / 4$ minute). Divide the ounces per minute by 128 , the ounces in a gallon, to get gallons per minute. This method is preferred to the other two methods described above because it gives an indication of nozzle flow uniformity.

The other two methods only give an average flow rate. If by chance half of the nozzles on a spray boom were delivering the equivalent of $20 \mathrm{GPA}$ and the other half 40 GPA, a tank refill test or a test using the graduations would indicate that the sprayer is applying the average of 20 and 40 or 30 GPA. The applicator would not know what the true conditions were. This hypothetical situation could not happen if the GPM is obtained from a nozzle flow test. Knowing that the nozzle flow rates are uniform is an absolute must for herbicide application because the tolerance of plants to excessive levels of herbicides is lower than for most of the other commonly used pesticides, but having uniform nozzle flow is desirable for any type of application.

Example. The flow from all 13 of the nozzles on a spray boom is caught for $1 / 2$ minute at each nozzle and the ounces caught at each is listed in Table 1 .
Table 1. Ounces water per $1 / 2$ minute flow on 13 nozzles of spray boom.

\begin{tabular}{|cc|cc||}
\hline Nozzle Number & Ounces & Nozzle Number & Ounces \\
\hline \hline 1 & 30 & 8 & 31 \\
2 & 32 & 9 & 29 \\
3 & 29 & 10 & 32 \\
4 & 31 & 11 & 30 \\
5 & 30 & 12 & 31 \\
6 & 30 & 13 & 29 \\
7 & 28 & & 182 total \\
\hline \multicolumn{4}{|l}{} \\
\hline
\end{tabular}

Grand total $=210+182=392$ ounces per $\_$min.;

Ounces per minute $=2 \times 392=784$;

Gallons per minute $=784 / 128=6.12 \mathrm{GPM}$

The table shows that the nozzles delivered various amounts. There is some variation in the flow delivered by new nozzles and often considerable variation among used ones. The amount of variation commonly considered acceptable is $+10 \%$ of the average amount. (Equation 6 )

$$
\text { Average }=\frac{\text { total flow }}{\text { no. of nozzles }}=\frac{392}{13}=30.2 \text { ounces }
$$

$10 \%$ of the average $=0.10 \times 30.2=3.02$ or 3.0

The acceptable range is found by subtracting 3.0 from the average and adding 3.0 to the average.

Acceptable range $=30.2+3.0=27.2$ to 33.2 ounces.

Any of the 13 nozzles that delivered between 27.2 and 33.2 ounces are acceptable. A nozzle with flows outside of this range should be replaced and the test rerun. All of the amounts caught from the 13 nozzles in this example fall within this range, so the set of nozzles on the boom is acceptable. If the ounces caught from any of the nozzles had fallen outside of the acceptable range, the flow from the nozzle would need to be rechecked before replacing it.

\section{Determining the Acres Treated per Minute}

The acres treated per minute of time depends on the treated swath width and the speed of the sprayer. The equation for determining acres/minute is: (Equation 7 )

$$
\text { Acres } / \text { minute }=\frac{\text { swath }(\mathrm{ft}) \times \text { speed }(\mathrm{ft} / \mathrm{min})}{43560}
$$

or (Equation 8 )

$$
\text { Acres } / \text { minute }=\frac{\text { swath }(\mathrm{ft}) \times \text { speed }(\text { miles } / \mathrm{hr})}{495}
$$

The swath is the width covered by the sprayer per pass through the field. The speed in feet per minute (fpm) can 
be determined in the field by setting two stakes 200 feet or more apart and measuring the time it takes to drive the distance between them when using the gear and engine RPM that will be used in the field. The first equation with speed in feet per minute is preferred to the second with speed in miles per hour because fpm can be accurately determined in the field. The speedometer on tractors pulling a sprayer or on self-propelled sprayers should not be used to determine the speed in mph because the accuracy of the speedometer depends on the tire size being the same as the original equipment tires, the tire inflation, and tire slippage. If the speed in miles per hour is needed for reference purposes, divide the speed in feet per minute by 88 since 1 mph equals $88 \mathrm{fpm}$.

If the spray is being applied to a field that has been plowed or harrowed in preparation for planting, the speed should be determined under actual field conditions to allow for tire slippage. This is especially true if a special incorporating tool or a harrow that exerts a high draft load on the tractor drawbar is used to incorporate the pesticide into the soil.

Example. A sprayer treats 10 rows of a crop planted on 3-foot rows. It takes 29 seconds to travel 200 feet when using the gear and RPM that will be used in the field. How many acres per minute are treated by the sprayer?

Swath is 10 rows $x 3 \mathrm{ft} /$ row = 30 feet ( Equation 9 );

$$
\text { Speed }=\frac{\text { feet }}{\text { minutes }}
$$

the 29 seconds must be expressed as minutes ( Equation 10 ).

$$
\text { Minutes }=\frac{29}{60}=0.48
$$

Speed $=200 / 0.48=417 \mathrm{fpm}$

( Equations 11,12)

$$
\text { Acres } / \text { minute }=\frac{\text { swath }(\mathrm{ft}) \times \text { speed }(\mathrm{fpm})}{43560}
$$

$$
\text { Acres } / \text { minute }=\frac{30 \times 417}{43560}=0.29
$$

(Equation 13)

$$
\mathrm{GPM}=\frac{\mathrm{GPM}}{\text { acres } / \mathrm{min}}
$$

A sprayer that delivers 6.12 GPM (the GPM determined from the nozzle flow test example) and covers 0.29 acres/ min would be applying 6.12/0.29=21.1 gallons per acre.
Determining the Acres Treated by a Volume of Spray

The number of acres treated by a sprayer depends on the volume of spray mixture in the tank and the gallons of spray applied per acre. The equation for determining the acres treated is: (Equation 14)

$$
\text { Acres treated }=\frac{\text { volume of spray mixed }}{\text { gal/acre }}
$$

The sprayer in the above example was applying 21.1 GPA. If 300 gallons of spray were mixed, it would treat $300 / 21.1=14.2$ acres.

A pesticide that is recommended on the label at 1 pint per acre would require 14.2 pints of pesticide in the tank and the remainder of the 300 gallons would be water or other diluent.

\section{Selecting Nozzles to Apply a Certain GPA}

The equation GPA = GPM/acres per minute is solved for GPM in order to choose the nozzles for the sprayer.

$$
\mathrm{GPM}=\mathrm{GPA} \times \mathrm{x} \text { acres per minute }
$$

Example. A grower wants to nozzle a sprayer to apply 50 gallons per acre. The sprayer treats five rows and the row spacing is 4 feet. The sprayer is equipped with nozzle drops in the row middles and five nozzles are directed at each row of plants (one above the row and two directed at the row from each side). Under the existing field conditions a safe speed was determined to be 572 feet per minute. Which hollow cone nozzles would apply the desired GPA rate at a pressure of 100 psi? (Equation 15 )

$$
\text { Acres } / \text { minute }=\frac{\text { swath }(\mathrm{ft}) \times \text { speed }(\mathrm{fpm})}{43560}
$$

Swath $=$ rows $\mathrm{x}$ row spacing $=5 \times 4=20 \mathrm{ft}$ (Equation 16)

$$
\text { Acres } / \text { minute }=\frac{20 \times 572}{43560}=0.263
$$

GPM required $=$ GPA $\times$ acres $/$ minute $=50 \times 0.263=$ 13.15

The total GPM required is 13.15. Since there are 25 nozzles on the boom ( 5 rows and 5 nozzles per row), each nozzle should deliver $13.15 / 25=0.526 \mathrm{GPM}$ at the $100 \mathrm{psi}$ pressure used.

Table 2 gives the GPM delivered by a series of hollow cone nozzles at various pressures. The nozzle consists of a nozzle body, a strainer, a core (whirl-plate), a disc (orifice), and a nozzle cap. In this example a nozzle that delivers close to the 0.526 GPM at 100 psi must be chosen. The 100 psi column of the table is entered and 0.54 GPM is 
Table 2. Performance tables of disc and core nozzles.

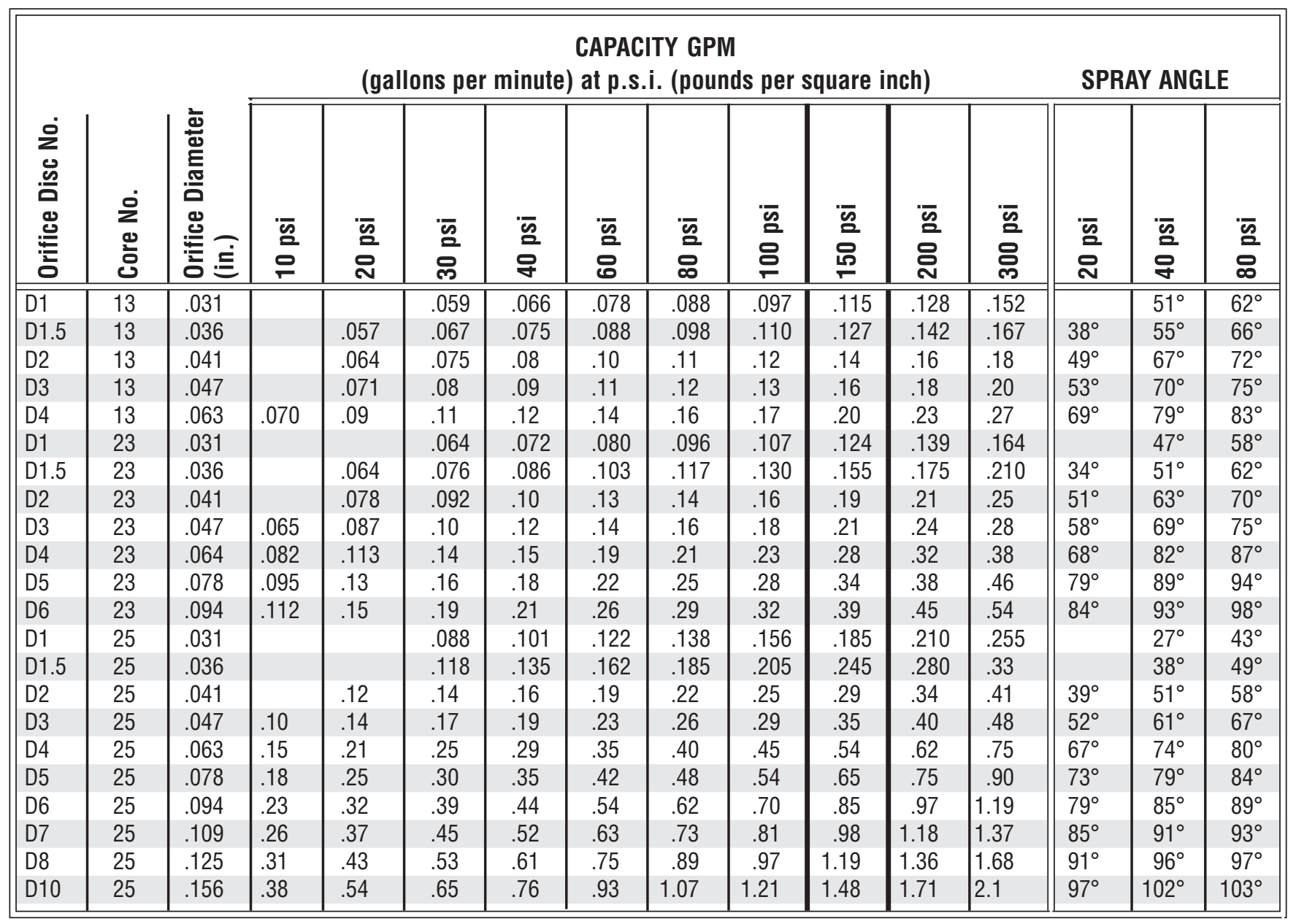


the closest to the desired 0.526 GPM. The 0.54 GPM is delivered by a D5 disc combined with a \#25 core. This combination would be referred to as a D5-25, disc and core nozzle. The D4-45 and the D3-46 combinations also deliver close to the desired GPM, but are not quite as close as the D5-25. If a grower bought new nozzles, D5-25s would be preferable; but, if a set of the other two combinations mentioned were already available, the grower could use them because they are close enough.

The GPM delivered by disc and core nozzles is the result of the combination of disc and core used. There were three nozzles in the example above that delivered close to the desired amount. They ranged from a relatively large orifice disc mated with a core having small holes (the D525 ) and a small orifice disc mated with a core having large holes (the D3-46).

There are other types of cone nozzles available in addition to the ones used in the example. Many of them are a single piece rather than the disc and core combination. The above example would be approached in the same manner regardless of what nozzles were being considered. The grower must choose a nozzle that delivers approximately $0.53 \mathrm{GPM}$ at whatever pressure is being used in the field.

\section{ALWAYS RUN A FIELD CALIBRATION TEST}

The example problem for selecting spray nozzles indicates that 25 nozzles delivering 0.526 GPM each would deliver 50 GPA at the pressure, speed, and swath assumed. A grower should never rely on mathematics alone to calibrate a sprayer. Mathematically determining the nozzles required is very helpful, but a field calibration (use the techniques described in the section on Calibrating a Nozzled Sprayer discussed earlier) must be conducted to determine the actual GPA applied by the sprayer.

Nozzle manufacturers' performance charts used to select nozzles give flow rates for new nozzles; the pressure is assumed to be at the nozzle and the liquid sprayed is assumed to be water. In contrast, the following may be true when calibrating a sprayer:

- Worn nozzles are used that deliver more than the chart values. (It is recommended that nozzles be replaced when the flow exceeds $10 \%$ more than delivered by new ones because the droplet spectrum and resulting coverage is adversely affected.)

- The spray pressure is read from a gauge that is inaccurate or is located at a point on the sprayer where the pressure is quite different from the pressure at the nozzles because of friction loss in sprayer hoses and fittings.
The spray solution flow characteristics could be significantly different from water. (This is not usually a problem in vegetable spraying with conventional ground sprayers because the spray is very dilute and usually flows like water.)

\section{APPLICATION RATES (GPA) OF DILUTED SPRAY ARE FLEXIBLE}

Assume that the field calibration test of the sprayer in the example above shows that the sprayer applies 44 GPA rather than the desired 50 GPA. A grower could choose between one of the three alternatives below:

Option 1. Change the pressure to increase the flow enough to apply $50 \mathrm{GPA}$. This is a reasonable choice in this case because the difference between the desired (50) and the measured (44) GPA is small. If the difference is large, it is not feasible to achieve the desired GPA by changing pressure. The flow rate varies as the square root of pressure, so large changes in pressure cause relatively small flow changes. As an example, the pressure has to be increased four times to double the flow. To determine the desired pressure (psi) that will produce the desired application rate (GPA) use: ( Equation 17 )

$$
\mathrm{PSI}_{2}=\mathrm{PSI}_{1} \times\left(\frac{\mathrm{GPA}_{2}}{\mathrm{GPA}_{1}}\right)^{2}
$$

The pressure that would make the sprayer that was using 100 psi pressure during the calibration test now apply 50 GPA is: ( Equation 18 )

$$
\mathrm{PSI}_{2}=100_{1} \times\left(\frac{50}{44_{1}}\right)^{2}=129 \text { psi }
$$

Option 2. Change speed to make the sprayer apply the desired GPA rate. The GPA rate varies indirectly with field speed of the sprayer. Doubling the speed will halve the GPA applied and halving the speed will double the GPA, if the nozzle flow remains constant. Changing speed is a good way to change the application rate. However, there is a practical limit to the change that can be accomplished by changing speed because going very slow requires too much time to complete the job and ? oing too fast for the field conditions can be dangerous. To determine the desired speed (FPM) that will give the desired application rate (GPA) use: (Equation 19)

$$
\mathrm{FPM}_{2}=\frac{\mathrm{GPA}_{1}}{\mathrm{GPA}} \times \mathrm{FPM}_{1}
$$


The speed that would make the sprayer that was traveling 572 FPM during the calibration test now apply 50 GPA is: (Equation 20 )

$$
\mathrm{FPM}_{2}=\frac{44}{50} \times 572=503 \mathrm{fpm}
$$

If the tractor or self-propelled sprayer has a tachometer to measure the engine speed in revolutions per minute (RPM), the easiest way to change the field speed of the sprayer is to leave the transmission in the same gear as it was when traveling 572 FPM and change RPM (in this case reducing RPM because the speed must be lower). To determine the desired RPM that will give a desired field speed (FPM) use: (Equation 21 ).

$$
\mathrm{RPM}_{2}=\frac{\mathrm{FPM}_{2}}{\mathrm{FPM}_{1}} \times \mathrm{RPM}_{1}
$$

Assume that the RPM of the sprayer was 2200 during the calibration test. The engine RPM that would make the sprayer speed 503 FPM rather than the 572 FPM traveled in the calibration test is: ( Equation 22 )

$$
\mathrm{RPM}_{2}=\frac{503}{572} \times 2200=1936
$$

In the equations 19-22:

PSI2 and PSI1 are the desired and measured pressures, respectively.

GPA2 and GPA1 are the desired and measured gallons per acre, respectively.

FPM2 and FPM1 are the desired and measured speed, in feet per minute, respectively.

RPM2 and RPM1 are the desired and measured tach readings in revolutions per minute, respectively.

Option 3. Apply the GPA that was determined during the calibration test. The amount of diluted spray applied per acre is usually not rigidly specified on the pesticide label, if specified at all. In the example problem the grower nozzled the sprayer to theoretically apply 50 GPA and the field calibration test showed it was actually applying 44 GPA. If the GPA of diluted spray determined by the calibration test is "reasonable" (within approximately $20 \%$ to $25 \%$ of the targeted amount) and the label does not forbid it, the grower could use the GPA "as is."

The amount of chemical formulation is recommended on the pesticide label within a relatively narrow range and a grower must adhere to the recommendation. The recommended amount of formulation can be applied regardless of the amount of diluted spray applied by tailoring the concentration of the formulation in the spray mixture.
Example. Determine the acres treated by a 200 gallon tank of spray when the GPA is 44 and 50: (Equation 23 ) or ( Equation 24 )

$$
\begin{aligned}
& \text { Acres } / \operatorname{tank}=\frac{200 \mathrm{gal} / \operatorname{tank}}{44 \mathrm{gal} / \mathrm{acre}}=4.54 \mathrm{acres} / \operatorname{tank} \\
& \text { Acres } / \operatorname{tank}=\frac{200 \mathrm{gal} / \mathrm{tank}}{50 \mathrm{gal} / \mathrm{acre}}=4.0 \mathrm{acres} / \operatorname{tank}
\end{aligned}
$$

A pesticide recommended at 3 pints per acre on the label would have $3 \times 4.54=13.62$ pints mixed in 200 gallons of spray, if 44 GPA were applied and it covered 4.54 acres. The same pesticide would only have $3 \times 4=$ 12 pints mixed in 200 gallons of spray, if 50 GPA were applied and it covered 4.0 acres. The amount of pesticide applied in both cases is 3 pints per acre in accordance with the label.

\section{WHAT PRESSURE SHOULD BE USED?}

The pressure used to apply pesticides depends on the pest problem. Low pressure (15 to $40 \mathrm{psi}$ ) is generally used for applying herbicides, medium pressure (80 to $100 \mathrm{psi}$ ) for applying insecticides, and high pressure (100 to 400 psi) is often used for applying fungicides.

Low pressure is generally used for applying herbicides with fan nozzles. The angle made by the edges of the nozzle fan depends on the nozzle design and the pressure. The most common fan angles are 80 and 110 degrees. The correct height of the nozzle tip above the sprayed surface when applying a broadcast application depends on the fan angle and the spacing of the nozzles on the sprayer boom ( Fig. 1). Pressures below 30 to 40 psi require special low pressure nozzles. The fan angle of conventional nozzles narrow down when the pressure is lower than $40 \mathrm{psi}$ and the pattern applied by the nozzles will not be uniform with the boom set at the standard height. Low pressure is used when applying herbicides to avoid drift of small droplets into neighboring fields or onto the planted crop when directed sprays are applied after the crop has emerged. Growers have adopted the use of low pressure when spraying herbicides because the results of herbicide drift, the killing or injury of nontarget plants, are so obvious.

Insecticides are applied at higher pressures than herbicides because the nozzles produce more droplets and plant coverage is usually better. Producing small droplets increases the potential for drift of insecticidal sprays, but the level of residue in neighboring areas that causes a noticeable problem is usually higher for insecticides than for herbicides.

Fungicides are sometimes applied at extremely high pressures (400 psi range). This high pressure is used pri- 


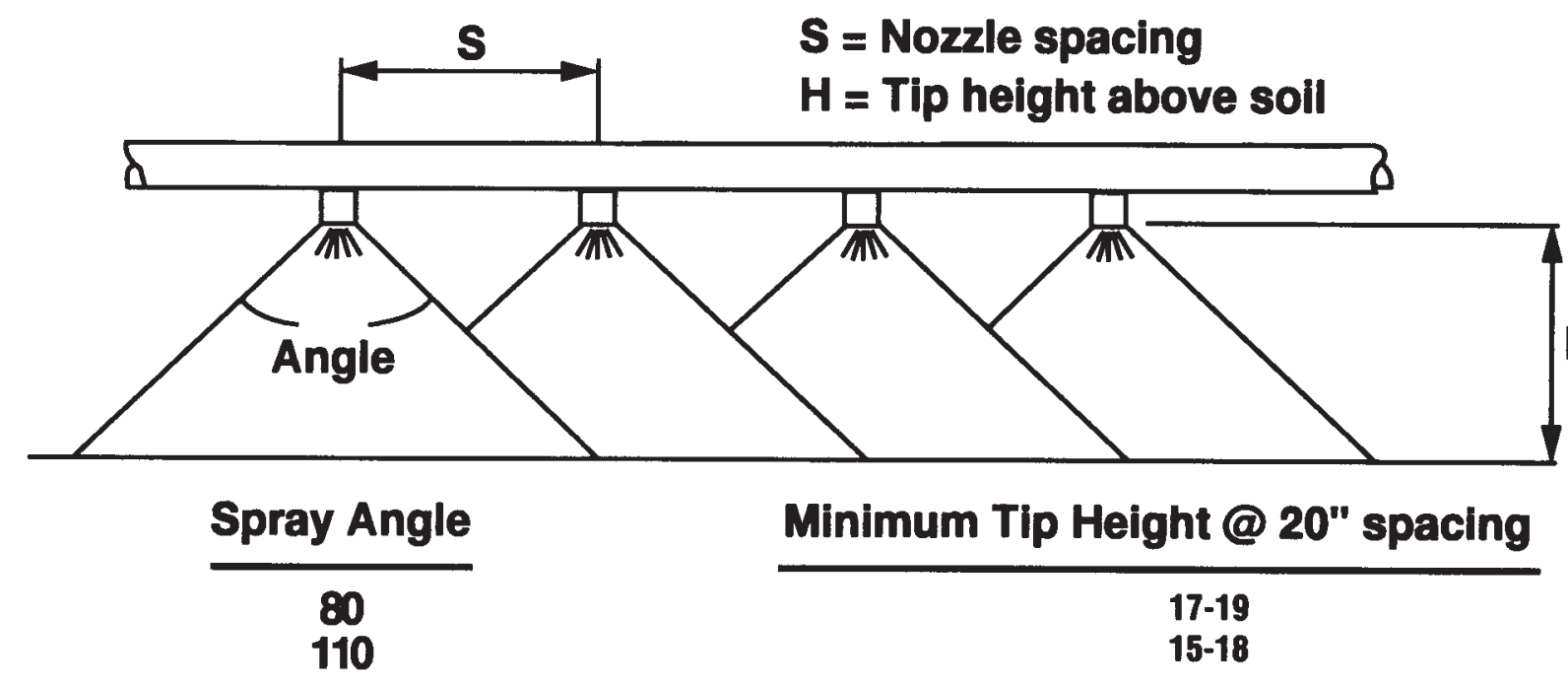

Fig. 1. Recommended heights of fan nozzles applying a broadcast application.

marily to improve coverage by producing a lot of small droplets. Using high pressure can improve coverage, but more of the small spray droplets drift from the field. Research has shown that many fungicides applied at relatively low pressure (50 to $100 \mathrm{psi}$ ) have yielded acceptable control of plant diseases. The primary exception being when treating a crop with fungicidal oils. Better disease control is obtained with oils using high pressure.

Even though the adoption of Integrated Pest Management (IPM) has kept the use of pesticides from expanding and growers are more aware of the causes of drift and how to control it, there is a good chance that there will be more drift problems in the future than in the past. Equipment is available that can detect much lower levels of chemical than was once possible. This equipment coupled with housing developments moving ever closer to long-standing agricultural operations in Florida and people who are anxious about the possible harm of low levels of chemicals in the environment should encourage growers to do all that is possible to avoid spray drift. There are many factors that influence drift other than spray pressure; but, generally speaking, the lowest spray pressure that yields acceptable coverage and control should be used.

\section{CALIBRATING APPLICATORS OF DRY MATERIALS}

Calibrating dry material applicators is very similar to the method described for sprayers. The basic equation used is: ( Equation 25 )

$$
P P A=\frac{P P M}{a c / m i n}
$$

where: PPA=pounds per acre;

PPM=pounds per minute;

$\mathrm{ac} / \mathrm{min}=$ acres per minute

The equation is good for any type of dry material application equipment ranging from band applicators mounted on a planter to aircraft. Dry material applicators often have manuals that give approximate settings of the opening in the hopper bottom that will apply the desired amount of various dry materials. These settings should only be used as a starting point to begin the calibration process. Generally, dry materials cannot be calibrated as accurately as liquids because the flow properties vary with humidity and the granules differ between batches.

\section{Determining Pounds per Minute Delivered}

Pounds per minute can be determined by 1) a hopper refill test; 2) by catching the amount delivered in a plastic bag; or 3) by catching the material on a tray mounted under a gravity flow drop spreader.

A hopper refill test is more suitable for applying nontoxic dry materials (fertilizer, etc.) because the granules are often scattered on the ground during the calibration process and cannot be retrieved. If spreading a toxic material during calibration poses a significant environmental problem, a large plastic sheet can be laid on the ground below the applicator and the material gathered after completing the calibration. It is also possible to get blank granules of the formulation used that do not have pesticide on them for conducting a calibration test. 
Catching the material in a bag during the calibration avoids the problem of having toxic granules in the area where the equipment is calibrated. This method is especially suited to calibrating application equipment used to apply bands of pesticide that are often centered on the crop row. A large bag can be placed over the bottom of a centrifugal spreader to catch granules, but care must be taken to keep the bag clear of the rotating disc that slings the granules over a wide swath.

Many manufacturers of drop spreaders offer a calibration tray that is mounted under the spreader outlet. The material falls on the tray during the calibration test rather than on the ground.

Hopper refill test. A dry material is poured into the hopper of the applicator and the material is leveled. After making a mark at the level of the material on the wall of the hopper, the applicator is run for 4 minutes. If it takes 36 pounds to restore the level in the hopper, how many pounds per minute are (PPM) applied? (Equation 26 )

$$
P P M=\frac{\text { pounds }}{\text { minutes }}=\frac{36}{4}=9 \text { pounds } / \mathrm{min}
$$

The methods described using the bag or the tray for gathering the material delivered by the applicator would again require dividing the pounds caught by the time that the calibration test lasted.
Determining the acres treated per minute. The same equation used for determining acres per minute treated by a sprayer is used for dry material spreaders. (Equation 27 )

$$
\text { Acres/minute }=\frac{\text { swath }(\mathrm{ft}) \times \text { speed }(\mathrm{fpm})}{43560}
$$

The swath treated by band applicators and drop spreaders can be determined by merely measuring the width that the material is observed on the ground under the equipment. A centrifugal spreader does not apply a uniform rate across the width treated by the spreader. The application rate lies between a triangular and a rectangular distribution. An approximation of the effective swath treated by centrifugal spreaders can be determined by measuring the distance between the outer edges where granules are observed to fall on each side of the spreader and take $75 \%$ of this distance as the effective swath ( Fig. 2 )

\section{Rate Per Acre Must Comply With Label}

When the pounds per acre determined from a calibration test of a dry material spreader differ from the application rate specified on the label, the grower must change the amount applied by changing speed or the size of the outlet in the bottom of the hopper until applications are close to the amount recommended on the label. Applying dry materials is less flexible than applying sprays. The concentration of dry materials is set at $10 \%, 50 \%, 80 \%$,

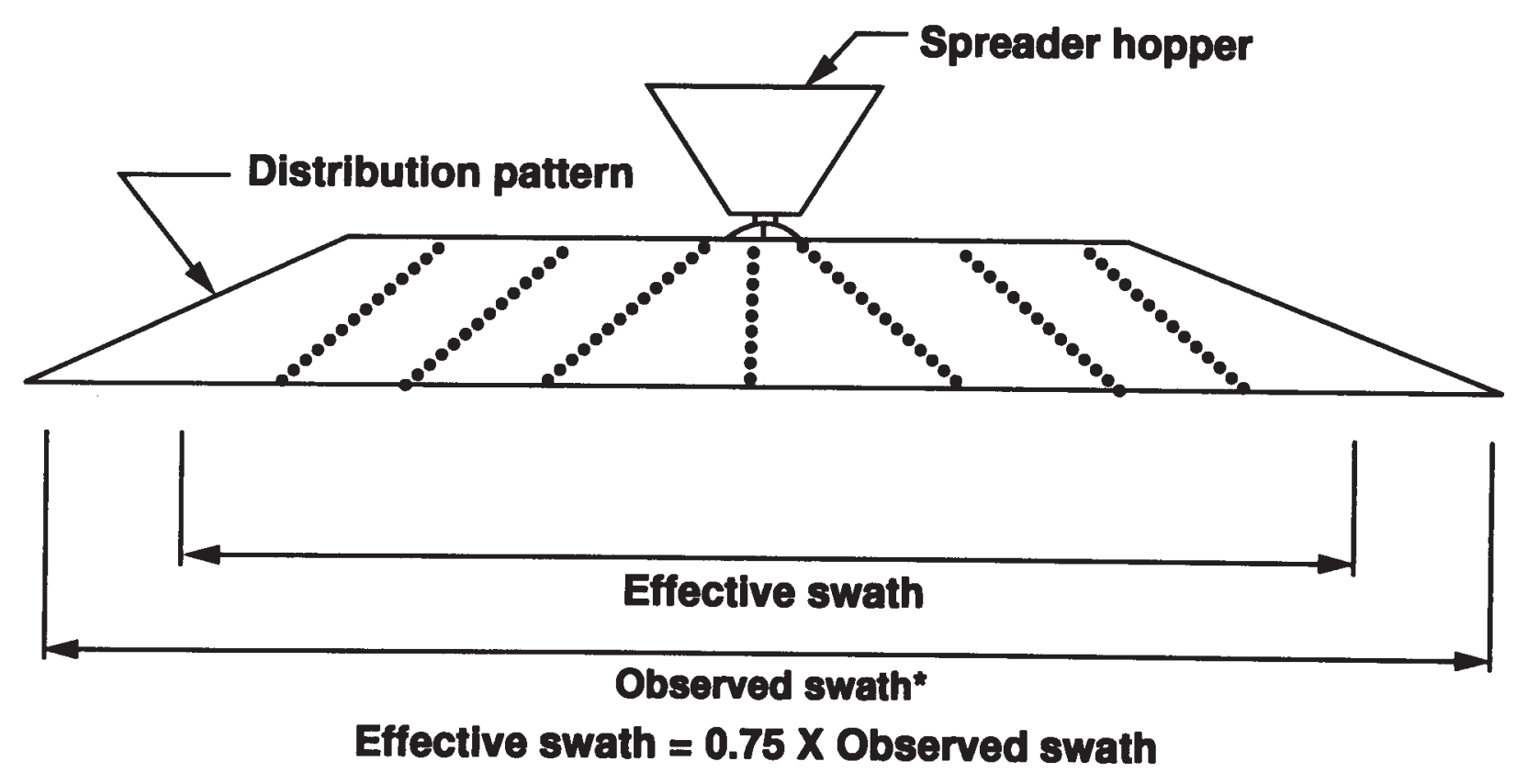

\section{* The observed swath is the distance between where the last granule is observed to fall on each side of the spreader.}

Fig. 2. The effective swath of dry materials spreaders. 
etc., when the material is formulated by the manufacturer. In the sprayer calibration portion of this publication, it was shown that the gallons per acre of diluted spray were somewhat flexible because the amount of chemical added to a tank load can be tailored to apply the amount of formulation specified on the label.

Example of dry material spreader calibration. A grower conducts a calibration test to determine the PPA applied by a centrifugal spreader. The amount of formulation caught in a bag after running the spreader for 2 minutes was 16 pounds. The speed in the gear and RPM to be used in the field was $430 \mathrm{fpm}$. Granules were observed to fall across a width of 38 feet. The material is recommended on the label at 25 pounds per acre.

1. What PPA is being applied?

2. What speed is required to make the unit apply the rate recommended on the label?

The pounds per minute delivered by the spreader and the acres per minute covered by the unit must be determined and the PPM divided by the acres/min. ( Equations 28,29 )

$$
\begin{gathered}
\mathrm{PPM}=\frac{\text { pounds caught in bag }}{\text { minutes }}=\frac{16}{2}=8 \\
\mathrm{Ac} / \mathrm{min}=\frac{\text { swath }(\mathrm{ft}) \times \text { speed }(\mathrm{fpm})}{43560}
\end{gathered}
$$

The distance that granules were observed to be thrown by the spreader was 19 feet on each side of the unit or a total of 38 feet. The distribution across the swath of a centrifugal spreader is not uniform. The application rate is usually maximum at the center and less at the ends of the swath. To compensate for the pattern variation the effective swath, which would be the swath spacing in the field, is computed to be $75 \%$ of the observed swath.
Effective swath $=0.75 \times 38=28.5$ feet (Equations 30, 31 )

$$
\begin{aligned}
& \mathrm{AC} / \mathrm{min}=\frac{28.5 \times 430}{43560}=0.28 \\
& \mathrm{PPA}=\frac{\mathrm{PPM}}{\mathrm{ac} / \mathrm{min}} \times \frac{8}{0.28}=28.6
\end{aligned}
$$

The label recommendation was for $25 \mathrm{PPA}$ and the measured application was 28.6 PPA. The speed will have to be increased for the PPA to decrease. The formula below gives the speed to apply the desired amount.

(Equations 32, 33 )

$$
\text { Desired speed }=\frac{\text { measured PPA }}{\text { desired PPA }} \times \text { measured speed }
$$

$$
\text { Desired speed }=\frac{28.6}{25} \times 430=492 \mathrm{fpm}
$$

$$
\text { or } 492 \div 88=5.6 \mathrm{mph} \text {. }
$$

A good way to increase the speed from 430 to $492 \mathrm{fpm}$ would be to leave the tractor in the same gear and increase the RPM by $492 / 430$ or 1.14 . If the RPM was 1800 during the initial calibration run, it should be increased to 2059 RPM (1.14 x 1800). A test would then be conducted to verify the speed by traveling between two stakes and dividing the distance by the time in minutes. 